\title{
Alternative splicing produces structural and functional changes in CUGBP2
}

\author{
Hitoshi Suzuki ${ }^{1,2^{*}}$, Makoto Takeuchi ${ }^{1 \dagger}$, Ayumu Sugiyama ${ }^{3,4}$, AHM Khurshid Alam², ${ }^{2,}$ Luyen Thi Vu ${ }^{2}$, \\ Yoshiharu Sekiyama², Hieu Chi Dam³,4, Shin-ya Ohki ${ }^{1,2}$ and Toshifumi Tsukahara 1,2
}

\begin{abstract}
Background: CELF/Bruno-like proteins play multiple roles, including the regulation of alternative splicing and translation. These RNA-binding proteins contain two RNA recognition motif (RRM) domains at the N-terminus and another RRM at the C-terminus. CUGBP2 is a member of this family of proteins that possesses several alternatively spliced exons.
\end{abstract}

Results: The present study investigated the expression of exon 14, which is an alternatively spliced exon and encodes the first half of the third RRM of CUGBP2. The ratio of exon 14 skipping product $(R 3 \delta)$ to its inclusion was reduced in neuronal cells induced from P19 cells and in the brain. Although full length CUGBP2 and the CUGBP2 $R 3 \delta$ isoforms showed a similar effect on the inclusion of the smooth muscle (SM) exon of the ACTN1 gene, these isoforms showed an opposite effect on the skipping of exon 11 in the insulin receptor gene. In addition, examination of structural changes in these isoforms by molecular dynamics simulation and NMR spectrometry suggested that the third RRM of R3 $\delta$ isoform was flexible and did not form an RRM structure.

Conclusion: Our results suggest that CUGBP2 regulates the splicing of ACTN1 and insulin receptor by different mechanisms. Alternative splicing of CUGBP2 exon 14 contributes to the regulation of the splicing of the insulin receptor. The present findings specifically show how alternative splicing events that result in three-dimensional structural changes in CUGBP2 can lead to changes in its biological activity.

\section{Background}

The CELF (CUGBP and ETR-3 Like Factor)/Bruno-like protein family plays important roles in the regulation of alternative splicing and translation [1-5]. In mammals, the CELF/Bruno-like family includes six members and is classified into two subgroups based on overall sequence similarity. One group is composed of CUGBP1 and CUGBP2, which share $76 \%$ amino acid sequence identity [6]. The other group contains BRUNOL1 (CELF3), BRUNOL5 (CELF5), BRUNOL6 (CELF6), and CELF4, which share $62-66 \%$ a.a. sequence identity with each other and $44 \%$ sequence identity with CUGBP1 [6]. CELF proteins have two consecutive RNA recognition motifs (RRMs) (RRM12) in the N-terminal region and another RRM (RRM3) in the C-terminal region. RRM2 and RRM3 are separated by

\footnotetext{
* Correspondence: suzuki-h@jaist.ac.jp

+ Contributed equally

${ }^{1}$ Center for Nano Materials and Technology, Japan Advanced Institute of

Science and Technology, Ishikawa 923-1292, Japan Full list of author information is available at the end of the article
}

a linker region that consists of $160-230$ amino acids. CELF family members are expressed in multiple tissues with a distinct tissue distribution pattern. CUGBP1 is expressed in almost all tissues, BRUNOL1 and BRUNOL5 are restricted to the brain, and CUGBP2 is abundant in the heart, skeletal muscle, and brain $[5,6]$.

The CELF family protein CUGBP1 was originally identified as an RNA-binding protein for CUG triplet repeats observed in the 3' UTR of the dystrophy myotonica protein kinase (DMPK) mRNA [7]. Increased copies of CUG triplet repeats from 5-37 to 50-5,000 cause myotonic dystrophy type I. CUGBP1 regulates the alternative splicing of exon 5 of cardiac Troponin T (cTNT) via the CUG repeats of muscle-specific enhancer elements (MSE) in its pre-mRNAs [1]. CUGBP1 is presumably involved in the incidence of DM because the splicing of $c T N T$ was disrupted in DM striated muscle. In addition to its role in the regulation of $c T N T$ exon 5 splicing, CUGBP1 is thought to be involved in controlling the alternative splicing of amyloid precursor protein $(A P P)$ [8], muscle-specific
C Biomed Central 
chloride channel $[9,10], \alpha$-actinin $(A C T N 1)[11,12]$, and the insulin receptor $(I R)[13,14]$. CUGBP2 (also known as ETR-3, Napor, and Brunol3), a paralogous protein of CUGBP1, is also known as an alternative splicing regulator. Similar to CUGBP1, CUGBP2 activates the inclusion of exon 5 in human $c T N T$ mRNA via binding to MSE $[3,15]$. CUGBP2 induces smooth muscle-specific exon inclusion via binding to uridine purine repeat elements (URE) in ACTN1 [11,12]. Although CUGBP2 is known as a splicing activator for the $\mathrm{N}$-methyl-D-aspartate receptor 1 (NMDA R1) exon 21, CUGBP2 represses NMDA R1 exon 5 inclusion [16]. In addition, CUGBP2 was reported to repress the inclusion of the IR exon 11 [17]. These studies showed that CUGBP2 has positive and negative regulatory roles in alternative splicing.

Bruno, another member of this protein family, is a translational repressor involved in germ cell formation in Drosophila [2]. The Bruno protein binds to the BRE (Bruno-responsive element) of the 3' UTR of oskar mRNA and represses its translation in the oocyte. The oskar protein is responsible for germ cell formation in the cytoplasm of the posterior pole of the oocyte. In vertebrates, EDEN-BP (embryo deadenylation element-binding protein), an orthologous protein of CUGBP1 in Xenopus, has been reported to control the translational regulation activity of specific maternal mRNAs via the EDEN sequence [18]. In addition, not only Bruno-like mRNA but also its protein, which is an orthologous protein of CUGBP1 in zebrafish, localized to the germplasm at the end of the cleavage furrow $[11,19,20]$. These results suggest that CUGBP1 is involved in translational control and germ cell formation.

The binding sequences of CUGBP2 and CUGBP1 can be classified into two groups. One group is represented by CUG triplet repeats of $c T N T, M t-P K, D M P K$ and $C /$ $E B P \beta[1,7,21]$, where the binding sequence essentially contains CUG repeats. Another group is represented by the BRE of oskar, EDEN of Eg5 and URE of ACTN1 $[2,11,18]$, and the binding sequence essentially contains uridine and guanine (UG)-repeats. Although there are three RRMs responsible for RNA-binding in CUGBPs, previous studies concluded that the RRM3 of CUGBPs binds to the UG-repeat in a sequence-specific manner [22]. Contradictory results showed that consecutive RRM1-2s bind to RNA in a sequence-specific manner. In addition to RRMs, the amino acids surrounding the RRMs were reported to affect RNA-binding and splicing activities. Indeed, RRM1-2 plus 70 residues of the adjacent downstream linker and RRM3 plus the last 119 amino acids of the adjacent upstream linker activated the MSE-dependent exon inclusion of $c$ TNT [15,17].

Most of the mammalian genes, including CUGBP2, are transcribed as alternatively spliced variants. Among the alternative exons of $C U G B P 2$, the present study focused on the skipping of exon 14, which encodes the first half of RRM3. Generally, an RRM is composed of two $\alpha$-helixes and four $\beta$-sheets $(\beta 1-\alpha 1-\beta 2-\beta 3-\alpha 2-\beta 4)$ formed by almost 80 a.a. [23]. The well-conserved RNP-1 and RNP- 2 correspond to the third $\beta$-sheet $(\beta 3)$ and first $\beta$ sheet ( $\beta 1)$, respectively. RNP-1 is important for the intercalation of target RNA in a sequence-specific manner [24]. Because exon 14 of the CUGBP2 gene encodes the first half of RRM3, a skipping transcript produces CUGBP2 truncated in parts of RRM3 (CUGBP2 R38). Similar to CUGBP2 R3 $\delta$, there are many proteins that encode unusual or partially truncated RRM domains in the database (Table 1). U2AF35 has an unusual RRM called the U2 homology motif (UHM) and its interaction with U2AF65 strengthens RNA binding $[25,26]$. Moreover, many proteins that contain partially deleted RRMs appear to be generated by alternative splicing. However, it is unclear how these proteins with partially truncated RRMs caused by alternative splicing function in biological processes.

The present study examined the expression patterns of CUGBP2 mRNA and its isoform, $R 3 \delta$, in P19 cells during neural differentiation and in different tissues. CUGBP2 was highly expressed in neural cells and in the adult brain compared with $R 3 \delta$, which was the major product in the kidneys, liver and undifferentiated P19 cells. Transient transfection experiments showed similar activities of CUGBP2 and R38, and both proteins promoted the use of the SM (smooth muscle) exon instead of the NM (nonmuscle) exon of the ACTN1 minigene. On the other hand, CUGBP2 and R3 $\delta$ had opposite effects on alternative splicing of exon 11 of $I R$; CUGBP2 repressed $I R$ exon 11 inclusion, whereas R3 $\delta$ did not and even slightly increased inclusion. This result suggests that the alternatively spliced isoform, R3 $\delta$, has a different function from that of CUGBP2. In addition, the results of molecular dynamics (MD) and NMR showed that the structure of the RRM domain differs significantly from that in the R $3 \delta$ isoform, resulting in the disruption of its binding activity.

\section{Results and discussion}

\section{Alternative splicing of CUGBP2}

A search of the UCSC genome browser, BLAT, suggested that the $R 3 \delta$ isoform is the product of skipping of exon 14 in $25 \%$ of CUGBP2 transcripts. Exon14 is $144 \mathrm{nt}$ and the skipping transcript does not generate a new premature termination codon. To assess in which organ alternative splicing of RRM3 in CUGBP2 takes place, RT-PCR was performed in adult mouse tissues. The products of exon 14 inclusion (CUGBP2) and exon 14 skipping (R3S), which encode a complete and partial RRM3, respectively, were detected (Figure 1A \&1B). CUGBP2 mRNA was highly expressed in the brain, where the main product was the exon 14 inclusion transcript encoding a complete 
Table 1 List of partial RRMs from the BLAST search

\begin{tabular}{|c|c|c|c|c|c|c|}
\hline Gene & Isoforms & Total RRMs & Short RRM & Lacking & Tandem & Alternative \\
\hline CELF4 & 4 & 3 & $3 r d$ & $\mathrm{~N}$-ter & RRM1-2 & Isoform 1/2/3 \\
\hline CPEB1 & 3 & 2 & 2nd & C-ter & RRM1-2 & - \\
\hline EIF3B & 1 & 1 & $1 s t$ & $\mathrm{~N}$-ter & - & - \\
\hline EIF4B & 1 & 1 & $1 s t$ & C-ter & - & - \\
\hline HNRPAB & 2 & 2 & 2nd & C-ter & RRM1-2 & - \\
\hline HNRPLL & 2 & 3 & $1 \mathrm{st}$ & C-ter & RRM1-2 & - \\
\hline HTATSF1 & 1 & 2 & 2nd & N- \& C-ter & - & - \\
\hline LEMD3 & 2 & 1 & $1 s t$ & $\mathrm{~N}$-ter & - & - \\
\hline LOC100132919 & 1 & 1 & $1 s t$ & C-ter & - & - \\
\hline NONO & 2 & 2 & $1 s t$ & $\mathrm{~N}$-ter & RRM1-2 & Isoform 1 \\
\hline RBM10 & 2 & 2 & $1 s t$ & $\mathrm{~N}$-ter & - & Isoform 1 \\
\hline RBM24 & 2 & 1 & $1 \mathrm{st}$ & $\mathrm{N}$-ter & - & Isoform 1/3 \\
\hline RBM28 & 2 & $3(4)$ & 1st (2nd) & C-ter & - & Isoform 1 \\
\hline RBM34 & 2 & $1(2)$ & $1 s t$ & C-ter & RRM1-2 & Isoform 1 \\
\hline RBMS1 & 2 & 2 & $1 s t$ & C-ter & RRM1-2 & - \\
\hline RBMS2 & 1 & 2 & $1 s t$ & C-ter & RRM1-2 & - \\
\hline RBMS3 & 1 & 2 & $1 \mathrm{st}$ & C-ter & RRM1-2 & - \\
\hline RDM1 & 8 & 1 & $1 s t$ & $\mathrm{~N}$-ter & - & Isoform $1 / 2 / 3 / 4$ \\
\hline ROD1 & 3 & 4 & $1 s t$ & C-ter & - & - \\
\hline RRP7A & 1 & 1 & $1 s t$ & C-ter & - & - \\
\hline SRSF1 & 2 & 2 & $1 s t$ & C-ter & - & - \\
\hline SSB & 1 & 2 & $1 s t$ & C-ter & - & - \\
\hline SYNCRIP & 6 & 3 & 2nd & C-ter & RRM1-3 & Isoform 1/2/5/6 \\
\hline TARDBP & 1 & 2 & 2nd & C-ter & RRM1-2 & - \\
\hline U2AF1 & 3 & 1 & $1 s t$ & $\mathrm{~N}$-ter & - & - \\
\hline U2AF2 & 2 & 3 & $3 r d$ & $\mathrm{~N}$-ter & RRM1-2 & - \\
\hline UHMK1 & 3 & 1 & $1 s t$ & $\mathrm{~N}$-ter & - & Isoform 3 \\
\hline
\end{tabular}

The list shows human proteins possessing an RRM with a deleted part selected from human RefSeq proteins. In 53 human Among 457 human RRM-type proteins, 53 human RefSeq proteins ( 27 human genes) showed a severely disrupted RRM domain. When two RRM domains have a short linker region (less than 50 a.a), these RRMs were considered as tandem RRMs. When an alternatively spliced isoform restored the complete RRM, the name of the isoform was listed. The parentheses show RRMs that were removed form the alternatively spliced isoforms.

RRM3 (Figure $1 C, R 3 \delta$ percentage of CUGBP2 and $R 3 \delta$ in the brain: $8.1 \%)$. The total amount of CUGBP2 transcripts in the kidney or liver was low compared to that in brain, but the percentage of the $R 3 \delta$ isoform was relatively high (Figure $1 C, R 3 \delta$ percentage of CUGBP2 and $R 3 \delta$ in the kidney: $22.1 \%$; in the liver, $19.5 \%$ ). These results suggest that the $R 3 \delta$ isoform is one of major products when their gene expression of CUGBP2 is low, and the CUGBP2 isoform, but not $R 3 \delta$, is expressed as the major product when their gene expression is high. To further investigate the alternative splicing pattern of CUGBP2, RT-PCR was performed in P19 cells during neural differentiation. Increased levels of the exon 14 inclusion product were detected during neural differentiation even though the exon 14 skipping product was not essentially changed (Figure 1D). The $R 3 \delta$ percentage on day 7 (neural cell stage) was $18.9 \%$, which was the lowest during P19 neural differentiation ( $R 3 \delta$ was $29.3 \%$ in undifferentiated P19 cells). The relatively low expression of $R 3 \delta$ with respect to alternative splicing patterns was also found in tissues of the adult mouse. Western blot analysis of CUGBP2 proteins showed that the full length isoform was the main protein in P19 cells and that its level increased at the neural stage. By contrast, the level of R3 $\delta$ decreased at the neural stage, although it was observed in undifferentiated P19 cells (Figure 1E).

\section{Expression analysis of CUGBP2 target genes, ACTN1 and insulin receptor}

CUGBP2 is a regulator of the alternative splicing of several transcripts, including ACTN1 [11] and IR [17]. The alternative splicing of $A C T N 1$ and $I R$ was therefore analyzed in adult mouse tissues and P19 cells. The ACTN1 gene has mutually exclusive exons, namely the smooth muscle (SM) exon and non-muscle (NM) exon (Figure 2A). The SM exon., as a percentage of NM and SM exons, was higher in neural differentiated P19 cells $(28.6 \%)$ than in undifferentiated P19 cells (6.7\%, Figure 2B). In the brain, $83.7 \%$ of ACTN1 transcripts contained the SM exon, while the NM exon was predominant in the kidney (SM exon: $26.6 \%$ ) 

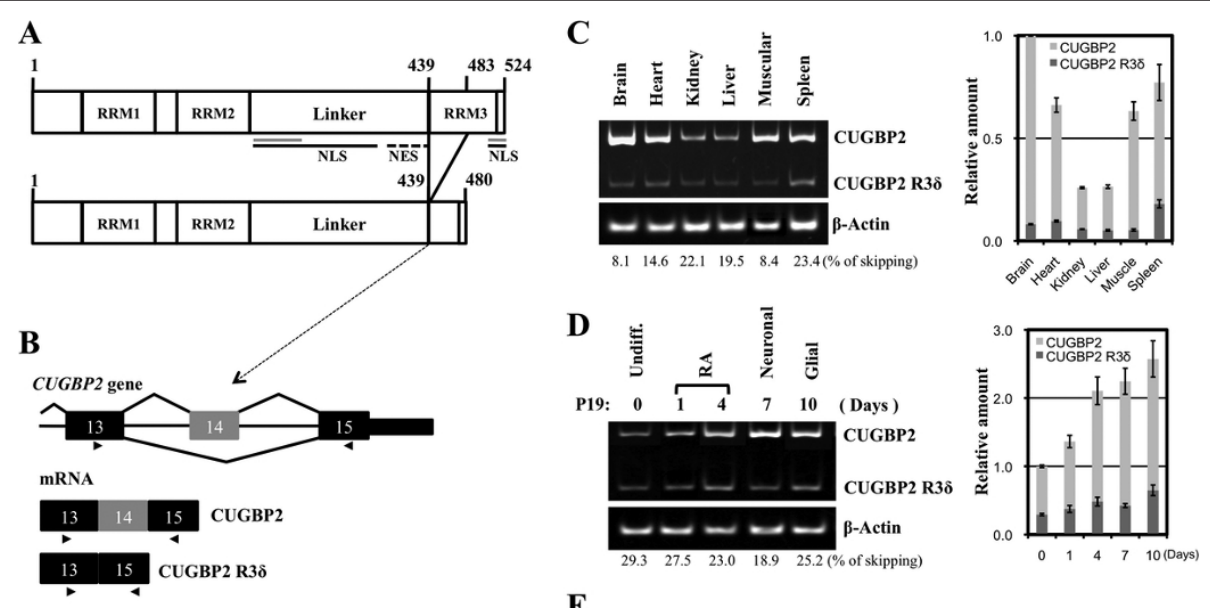

$\mathbf{E}$

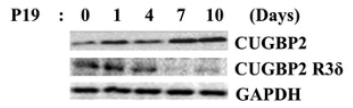

Figure 1 Alternative splicing of the CUGBP2 gene. (A) Schematic representation of the CUGBP2 protein and the CUGBP2 R3 $\delta$ isoform. The upper panel shows CUGBP2 and its domains. RRMs represent the RNA-binding domains. The NLS (line), NES (broken line), and splicing activation domain (gray line) were determined in a previous report [27]. The lower panel shows the alternatively spliced form of CUGBP2, the R3 $\delta$ isoform. (B) Schematic representation of exon 14 and its adjacent region in the CUGBP2 gene. Exons are indicated as black boxes with the alternatively spliced exons indicated as gray boxes. Introns are indicated by a central narrow line. Arrows show primer sites. (C) Expression analysis of CUGBP2 in adult mouse tissues. Semi-quantitative RT-PCR was performed using primers to detect the alternatively spliced exon of CUGBP2. The right side indicates the positions of exon 14 skipping or inclusion products. $\beta$-Actin was used as a control. (D) Expression analysis of CUGBP2 in P19 neural differentiation. The right side indicates the positions of exon 14 skipping or inclusion products. $\beta$-Actin was used as a control. Relative amounts of exon 14 skipping and inclusion products were estimated by densitometry. Changes of total expression levels were normalized using brain samples (C) or Day 0 samples (D). The error bars indicate the standard error. The values under the gel images indicate the percentage of the exon 14 skipping in total CUGBP2 transcripts. (E) Western blot analysis of CUGBP2. Whole cell extracts of P19 cells $(2 \mu \mathrm{g})$ were used to detect the changes in the amount of full-length CUGBP2 in the upper panel. The middle panel shows the R3 $\delta$ isoform detected using $7 \mu \mathrm{g}$ of each extract. GAPDH was used as a control and is shown in the lower panel.

and liver (Figure 2C, SM exon: 7.6\%). CUGBP2 has been suggested to promote the inclusion of the SM exon in prior work [11,12], suggesting that the elevated expression of CUGBP2 and inclusion of the ACTN1 SM exon may occur in the same cells and tissues.

On the other hand, the $I R$ gene consists of 22 exons and generates 2 isoforms, IR-A and IR-B, which are characterized by the skipping and inclusion of exon 11, respectively (Figure 2A) [28]. RT-PCR analysis of the alternative splicing of the $I R$ gene in tissues and P19 cells identified the two isoforms, IR-A and IR-B, in P19 cells during neural differentiation (Figure 2B). Although the expression of the $I R$ gene increased during the glial cell stage, the IRA/IR-B ratio did not change significantly during cell differentiation (the IR-A form, as percentage of IR-A and IR-B, was $86.4 \%$ in undifferentiated P19 cells and $80.7 \%$ in neural differentiated P19 cells). While the exon 11 skipping variant IR-A was expressed in the brain (IR-A: 87.6\%), the exon 11 inclusion form IR-B was mainly expressed in the kidney (IR-A: $4.3 \%$ ) and liver (IR-A: $5.0 \%$ ). Because CUGBP2 is known to repress the inclusion of $I R$ exon 11 [17], elevated expression of
CUGBP2 and the IR-A variant may occur in the same tissues, but is not observed in P19 cells. In addition to the expression of $C U G B P 2$, the ratio of $R 3 \delta$ to CUGBP2 was lower in the brain than in the kidney and liver. The function of the $R 3 \delta$ isoform was therefore examined in detail as described in the next section.

\section{CUGBP2 R3 $\delta$ induces smooth muscle type splicing of ACTN1}

As described previously, CUGBP2 promotes the inclusion of the mutually exclusive SM exon instead of the NM exon in ACTN1 [11,12]. The specific function of the CUGBP2 isoform R3 $\delta$ was examined by transient transfection using the mouse ACTN1 minigene (Figure 3A). Transfection of COS7 cells with the ACTN1 minigene alone resulted in the detection of a transcript including the NM exon as a major product and another transcript with the SM exon as a minor product (Figure 3B). Control COS7 cells lacking the minigene did not show these transcripts, confirming that the two transcripts containing the NM or SM exon were products of the transfected minigene. Co-transfection with a myc-tagged CUGBP2 

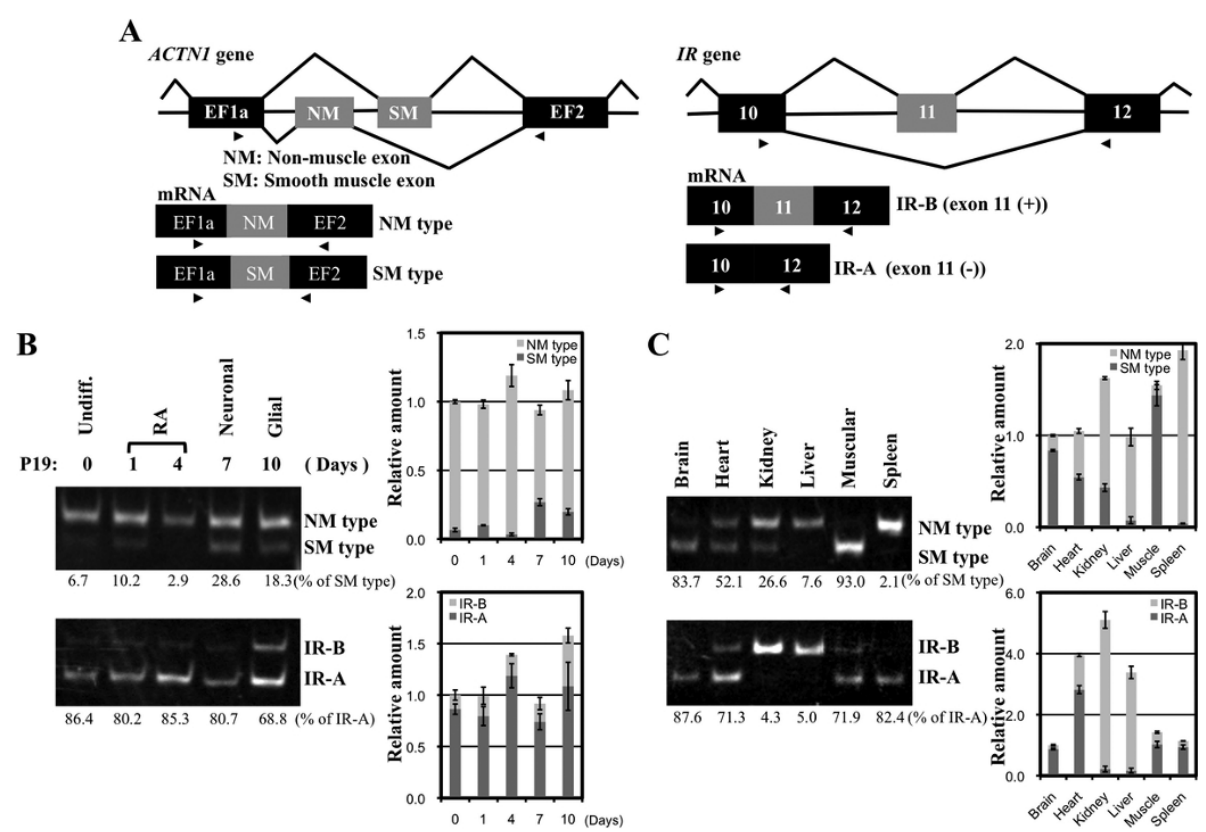

Figure 2 Expression analysis of the alternative splicing of the ACTN1 and IR genes. (A) Schematic representation of the mutually exclusive splicing of the ACTN1 and IR genes. The genomic structure and alternatively spliced mRNAs of ACTN1 are shown in the left panel and those of $\mathbb{I}$ in the right panel. Exons are indicated as black boxes with alternatively spliced exons depicted as gray boxes. Introns are indicated with a central narrow line. The arrow indicates the primer sites. (B) Expression analysis of ACTN1 and IR in P19 neural differentiation. Semi-quantitative RT-PCR was performed using primers to detect the alternatively spliced exons as shown in Figure 2A. The right side shows the positions of the SM exon and NM exon products or exon 11 skipping and inclusion products. (C) Expression analysis of ACTN1 and IR in adult mouse tissues. Semi-quantitative RT-PCR was performed using primers to detect the alternatively spliced exons. The right side shows the positions of the alternatively spliced products. $\beta$-Actin is shown as a control in Figure 1. The relative amounts of each PCR product were estimated by densitometry. Total expression levels were normalized using Day 0 samples (B) or brain samples (C). The error bars indicate the standard error. The values under the gel images indicate the percentage of the SM type or IRA in total ACTN1 transcripts or IR transcripts.

expression vector resulted in a decrease in the NM exon product concomitant with an increase in the SM exon product (Figure 3B \& Additional file 1: Figure S1). Use of the Etr-1 expression vector as a control showed that Etr1 promoted the use of the NM exon. These results are consistent with previous reports [11].

To assess the function of the alternatively spliced isoform of CUGBP2 R3 8 , transient transfection experiments were performed using the $A C T N 1$ minigene with the R $3 \delta$ instead of the CUGBP2 expression vector. $\mathrm{R} 3 \delta$ induced the production of the mutually exclusive SM exon similar to CUGBP2 (Figure 3B \& S1), suggesting that the function of R3 $\delta$ in $A C T N 1$ splicing is similar to that of CUGBP2 and that part of RRM3, which is encoded in $\mathrm{R} 3 \delta$, may be dispensable for the regulation of the splicing of ACTN1. As shown above, our results identified the brain as the location of SM exon inclusion and the kidney and liver as characteristic of NM exon inclusion. In parallel to SM exon inclusion, the expression level of $C U G B P 2$ was high in the brain and low in the kidney and liver, suggesting that the upregulation of CUGBP2 in the brain contributes to the induction of SM exon inclusion.

\section{Different effects of CUGBP2 isoforms on the insulin receptor}

It was reported that CUGBP2 induces the skipping of exon 11 in $I R$ [17]. The induction of exon 11 skipping by CUGBP2 was assessed using the $I R$ minigene in transient transfection experiments (Figure 3D). Transfection of COS7 or HeLa cells with the IR minigene alone resulted in the production of similar amounts of exon 11 inclusion and skipping products (Figure 3E). Exon 11 inclusion and skipping products were not detected in COS7 or HeLa cells without the minigene, confirming that they were transcribed from the transfected minigene. Cotransfection of the CUGBP2 expression vector and the $I R$ minigene caused an increase in the exon 11 skipping product and a decrease in the exon 11 inclusion product (Figure 3E \& S1). Co-transfection of Etr-1 as a control did not cause significant changes compared with the transfection of the minigene alone (Figure 3E \&3F). These results confirmed that CUGBP2 induces the skipping of exon 11, as previously reported. This effect was clearer in HeLa cells than in COS7 cells (Figure 3E, data not shown). 

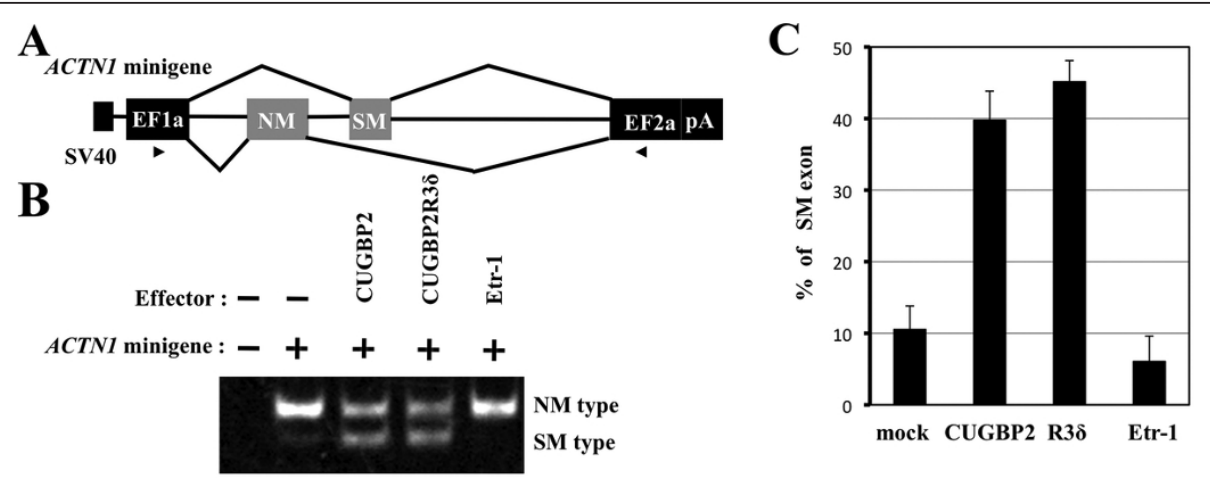

D
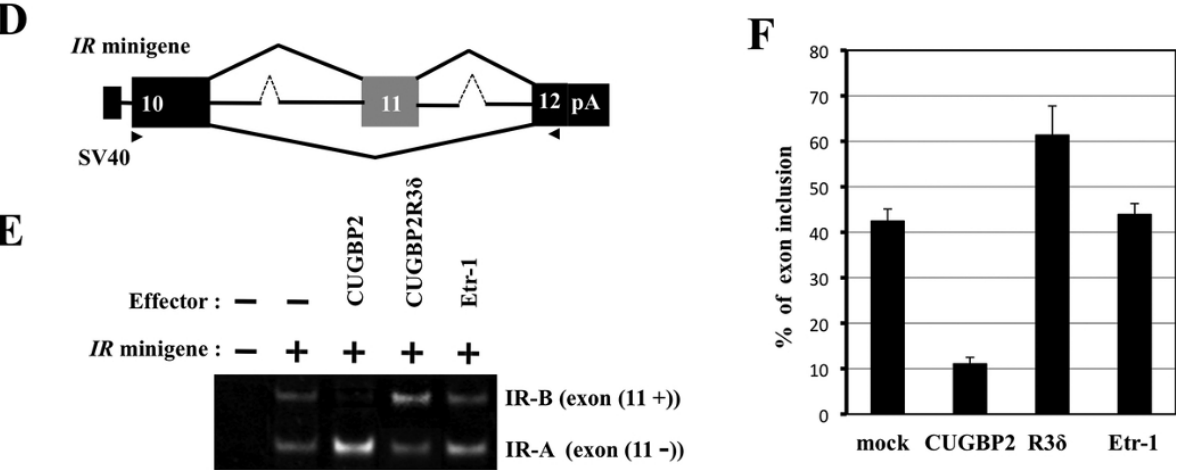

Figure 3 Transient transfection of the ACTN1 minigene and the $I R$ minigene. (A) Schematic representation of the ACTN1 minigene. Exons are indicated as black boxes with alternatively spliced exons indicated as gray boxes. Introns are indicated with a central narrow line. The arrows show the primer sites. (B) Results of the transient transfection experiment. COS7 cells were transiently transfected with the ACTN1 minigene with/without the CUGBP, CUGBP2 R38 isoform, or Etr-1 expression vectors. Alternatively spliced products were analyzed by RT-PCR. (C) Densitometric analysis of the transfection products. Quantification of the alternatively spliced products was performed by densitometry. The percentage of SM exon inclusion with respect to total product is shown in graphical representation. The error bars indicate the standard error. (D) Schematic representation of the $\mathbb{R}$ minigene. Exons are indicated as black boxes and alternatively spliced exons are indicated as gray boxes. Introns are shown with a central narrow line. The arrows show the primer sites. (E) Results of the transient transfection experiment. HeLa cells were transiently transfected with the IR minigene with/without the CUGBP, R38 isoform, or Etr-1 expression vectors. Alternatively spliced products were analyzed by RT-PCR. (F) Densitometric analysis of the transfection products. Quantification of the alternatively spliced products was performed by densitometry. The percentage of exon 11 inclusion with respect to total product is shown in graphical representation. The error bars indicate the standard error.

To examine potential changes in the function of the alternatively spliced isoform, $\mathrm{R} 3 \delta$, the $I R$ minigene was co-transfected with the R3 $\delta$ expression vector, which caused a decrease in exon 11 skipping and an increase in exon 11 inclusion in comparison to co-transfection with CUGBP2 or the mock control (Figure 3E \& 3F). In a previous report, artificially truncated CUGBP2 proteins with deleted $\mathrm{N}$-terminal regions or deleted $\mathrm{C}$-terminal regions were unable to engage in efficient exon 11 skipping [17]. In the present study, $\mathrm{R} 3 \delta$ promoted the exon 11 inclusion, which is in contrast to the activity to full-length CUGBP2, which promotes exon 11 exclusion of the $I R$ gene. These results also indicate that part of RRM3, which is encoded by the alternative exon (exon 14) of $C U G B P 2$, is not only essential for the skipping of $I R$ exon 11, but that disruption of this part of RRM3 may result in a change in the splicing regulated by CUGBP2. Among CELF family proteins, an isoform resembling $\mathrm{R} 3 \delta$ can be predicted in CELF4 (Table 1). However, a generalized rule for RRM3s cannot be established because exon 11 skipping can be activated by a subgroup of proteins that includes CUGBP1 and 2, but not by another subgroup that includes Etr-1 (CELF3) or CELF4 (Figure 3E) $[17,29]$. Our results suggest that the disruption of part of RRM3 in CUGBP2 generates a new activity in the regulation of the splicing of the $I R$ gene. Because the R $3 \delta$ isoform has a similar activity to CUGBP2 in the regulation of splicing of ACTN1, it cannot be considered simply as a dominant negative isoform of CUGBP2. The product of exon 11 skipping was detected in the brain and the product of exon 11 inclusion was detected in the kidney and liver, suggesting that high CUGBP2 expression in the brain contributes to exon 11 skipping. A high ratio of $R 3 \delta$ to $C U G B P 2$ in the kidney and liver may repress exon 11 skipping due to the specific function of the R $3 \delta$ isoform. 
Structural analyses of the third RRM of the CUGBP2 R3 $\delta$ isoform

A total of 457 human RefSeq proteins with RRM domain(s) were found in the BLAST. An RRM domain is usually $70 \sim 80$ a.a. in length, and slightly smaller RRM domains ( 60 a.a), which appear to lack rnp-2 in the $\mathrm{N}$-terminus or the dimerization module in the $\mathrm{C}$ terminal region, are frequently observed in conserved domain searches of BLAST. RRM3 of CUGBP2 consists of 75 a.a., and 44 a.a. of this region are disrupted in the R3 $\delta$ isoform. Disruption of almost half of RRM did not prevent recognition of the remaining sequence in the conserved domain search. A total of 53 human RefSeq proteins (27 human genes) with severely disrupted RRM domains are listed in Table 1 . Some of the domains shown in Table 1 such as eIF3B, eIF4B, La (SSB), U2AF35 (U2AF1) and U2AF65 (U2AF65), have been reported to form unusual RRM structures, although the amino acid sequences were not sufficient to predict specific RRM structures [26,30-32].

There-dimensional structures of the RRM3 isoforms were examined by MD simulation and NMR spectroscopy. The structural analysis of the RRM3 of CUGBP1 has been reported previously [22]. Several unaligned residues were found between the RRM3s of CUGBP1, and 2. The results of homology modeling showed that the third domain of CUGBP2 formed an RRM structure (Figure 4A). No significant difference could be observed between the MD simulations of RRM3 of CUGBP1 and RRM3 of CUGBP2. (data not shown). Further, CUGBP2 RRM3 maintained an RRM structure for $5 \mathrm{~ns}$ in the MD simulation (Figure 4B). The ${ }^{1} \mathrm{H}_{-}{ }^{15} \mathrm{~N}$ HSQC spectrum of the RRM3 of CUGBP2 in the absence of RNA showed that, unlike RRM3 of CUGBP1, the resonances of the $\mathrm{N}$-terminal residues in the linker domain were concentrated in the center of the spectrum, suggesting a random coil structure. This result was probably due to the inclusion of a long $\mathrm{N}$-terminal sequence in the present constructs, which forms a flexible linker region connecting RRM2 with RRM3 (Figure 5A).

Protein threading and comparative modeling of the $\mathrm{R} 3 \delta$ isoform were performed in the PDBj. The RRM3 of human CUGBP1 was chosen as the ideal template in the search and it was predicted that the $\mathrm{R} 3 \delta$ isoform with part of the linker sequence would not form an RRM structure, especially as the first $\alpha$-helix $(\alpha 1)$ was
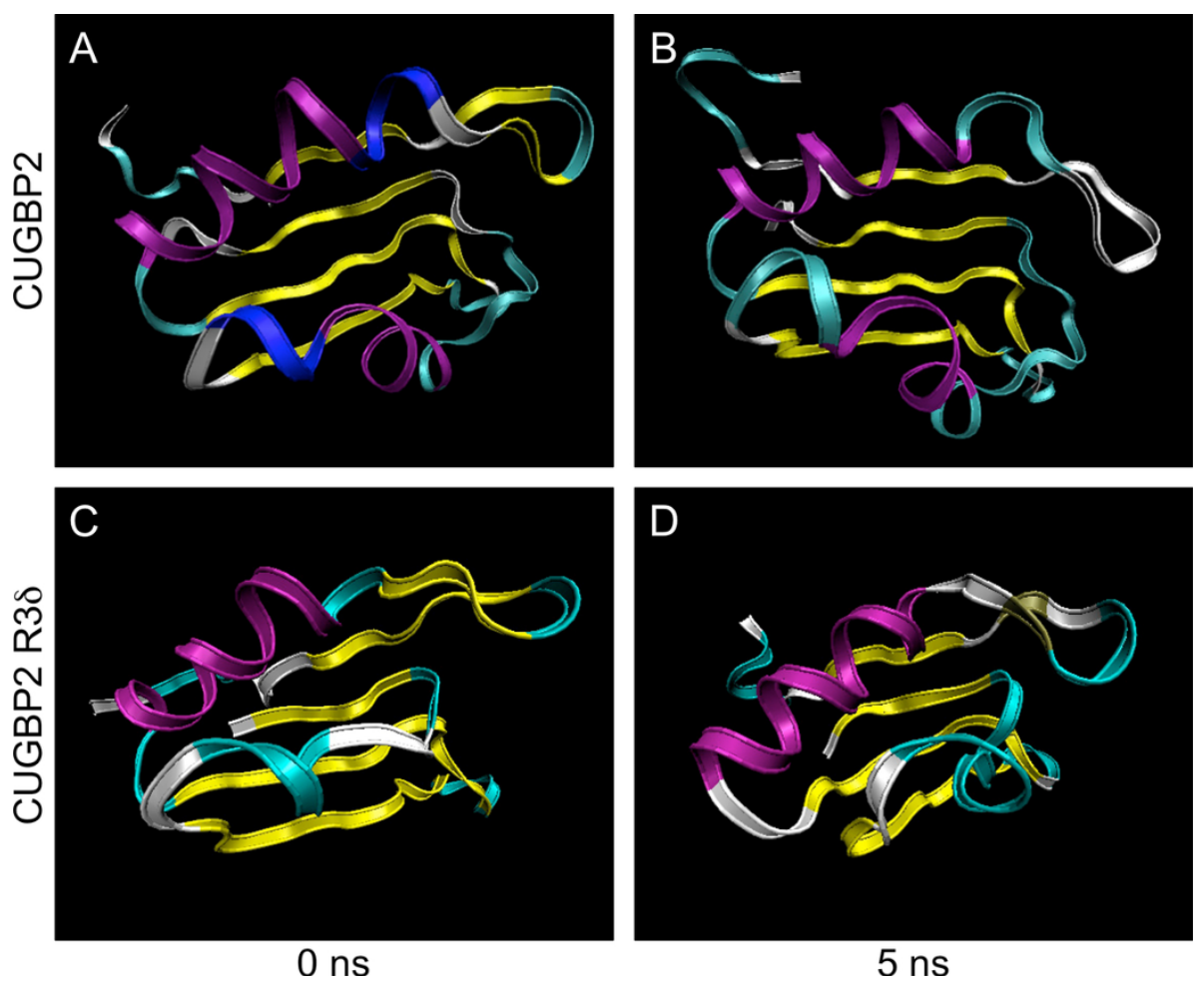

Figure 4 Structure transitions of the CUGBP2 and R3 $\boldsymbol{\delta}$ isoforms. (A) Initial structure of the CUGBP2 RRM3. The structure was predicted using CUGBP1 RRM3 (2rq4a) because the amino acid sequences of RRM3 of CUGBP1 and 2 are almost identical. (B) MD simulation of the CUGBP2 RRM3. MD simulation was performed for $5 \mathrm{~ns}$. (C) Initial structure of the R3 $\delta$ isoform RRM3. The RRM3 and the linker residues of the R3 $\delta$ isoform were analyzed by comparative modeling by SFAS in PBDj. (D) MD simulation of the R3 $\delta$ isoform. The result of comparative modeling was used for $\mathrm{MD}$ simulation for 5 ns to analyze the folding and stability of the predicted structure. 


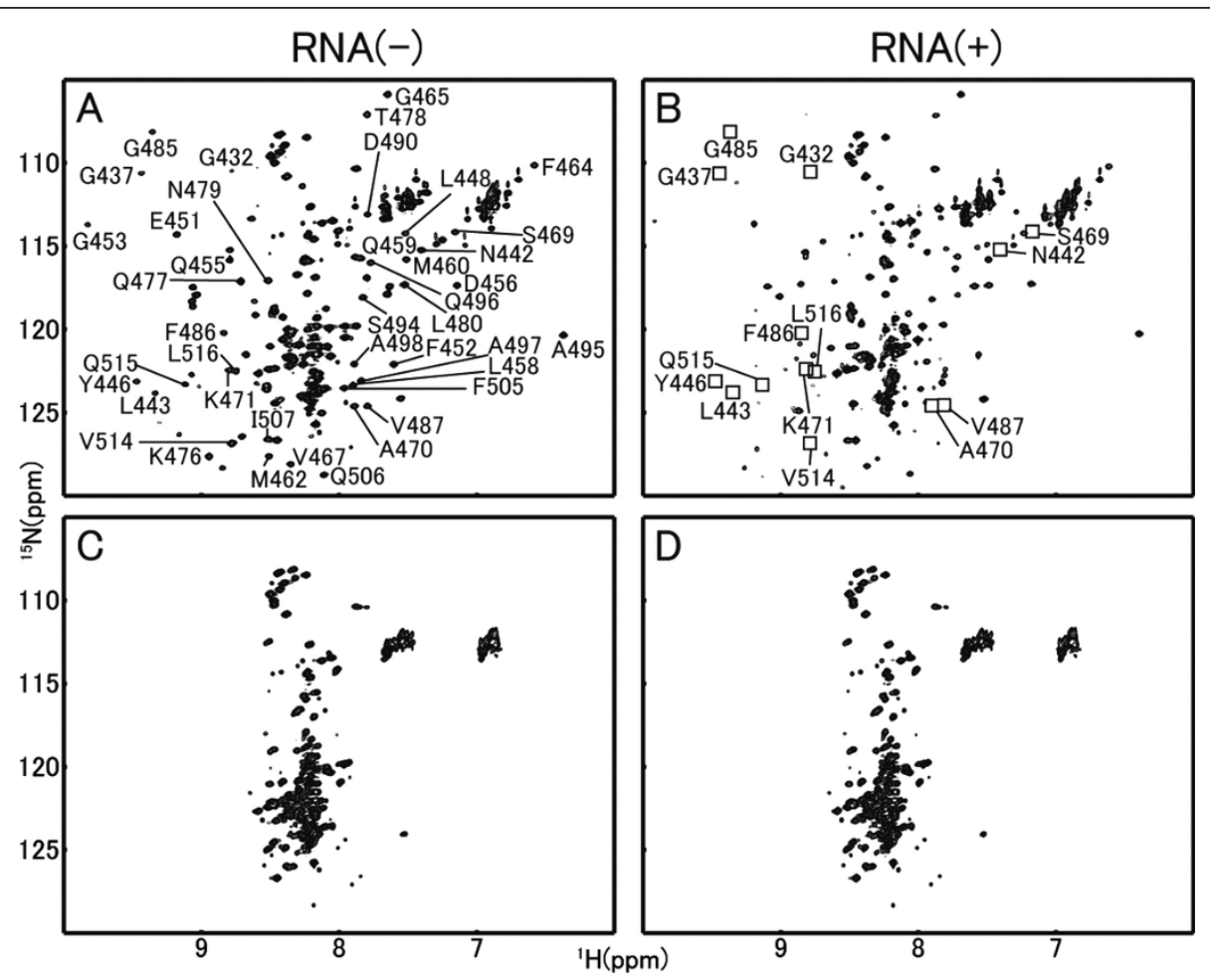

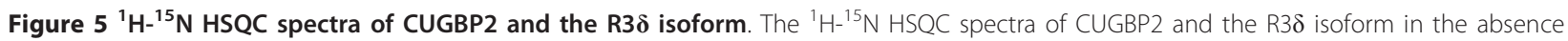
(left) and presence of RNA (protein:RNA = 1:1, right). For clarity, only certain resonances are labeled with one-letter amino acid codes and numbers [for further details, see Tsuda et al., 2009] [22]. The open boxes of panel B highlight the resonances that disappear with the addition of (UG)3 RNA

missing (Figure 4C). We used MD simulations to investigate whether the addition of linker residues to the RRM domain could restore its structure. However, even in these conditions, the first $\alpha$-helix cannot be formed even after 5 ns (Figure 4D). Moreover, resonances corresponding to the R3 $\delta$ isoform were detected in the central region of the NMR spectrum, suggesting the presence of greater alterations in the structure of R3 $\delta$ than those shown by MD simulation (Figure $5 \mathrm{C}$ ) and indicating that the RRM structure cannot be restored in the $\mathrm{R} 3 \delta$ isoform. In addition, the interaction between CUGBP2 or the R3 $\delta$ isoform and RNA molecules was examined. The addition of RNA caused the disappearance of many resonances and the appearance of new resonances in CUGBP2 (Figure 5B). The affected residues, which are highlighted with an open box in Figure 5B, were consistent with the corresponding region of CUGBP1 [22] and indicate that CUGBP2 binds to (UG) ${ }_{3}$ in a similar manner as CUGBP1. No spectral changes were detected in the R3 $\delta$ isoform with the addition of RNA (Figure 5D), indicating that the R3 $\delta$ isoform does not bind to (UG) ${ }_{3}$.

Although the RNA-binding of RRM1-2 of CUGBPs is still unclear, RRM3 is known to be responsible for RNAbinding to UG-repeats [22]. MD simulation suggested that the R3 $\delta$ isoform could not form an RRM domain and the NMR spectrum showed that it did not bind to the UG-repeat. These results lead to the speculation that the disruption of the first half of RRM3 by exon 14 skipping causes a defect in RNA-binding. However, the R3 $\delta$ isoform may interact with $A C T N 1$ pre-mRNA based on the fact that this isoform still has two RRMs and two different $c i s$-elements were reported in the alternative splicing of ACTN1 [11,12]. A CUGBP1 interacting ciselement was determined in the intronic region of $I R$ and shown to affect the alternative splicing of $I R$ [33]. Because this sequence is not a typical UG-repeat, perhaps the RRM3 may not be responsible for the primary interaction with the $I R$ pre-mRNA. Therefore, other, currently unknown, proteins might interact with the RRM3 domain and it might be the interactions of these proteins rather than RRM3 RNA-binding activity that is abrogated by RRM3 alternative splicing.

\section{Conclusion}

The results of the present study show that while CUGBP2 and its R3 $\delta$ isoform similarly promoted the use of the SM exon instead of the NM exon in the alternative splicing of the ACTN1 minigene, they did not similarly promote the use of exon 11 in the alternative splicing of the IR minigene; CUGBP2 promoted skipping of exon 11, 
whereas R3 $\delta$ did not. In addition, the results of MD simulation and NMR suggested that the truncated RRM3 region in $\mathrm{R} 3 \delta$ resulting from the alternative splicing of CUGBP2 could neither form a new RRM domain nor bind to a UG-repeat. Thus, it is possible that other, currently unknown, CUGBP2-interacting proteins and/or other splicing factors involved in splicing regulation might be important for RRM3 function. Our results also showed that the skipping of exon 14 of CUGBP2 not only disrupted CUGBP2 RNA-binding activity but also altered its splicing regulator function. A high ratio of $R 3 \delta$ to $C U G B P 2$ in the kidney and liver may affect the splicing of the $I R$ gene and repress exon 11 skipping.

\section{Methods}

\section{Plasmid construction}

The entire open reading frame of CUGBP2 cDNA (NM_001110228.1) and its alternatively spliced isoform, CUGBP2 R3 $\delta$ (NM_010160.2), were amplified by RT-PCR using mouse brain total RNA and the following primers: TGTACTCGAGATGCGCTGTCCCAAATCC and TCTG TCTAGAGGATCAGTAAGGTTTGCTGTCG. The resulting cDNAs were subcloned into the pCS2+ MT vector using Xho I and Xba I sites. RRM3 and RRM3 R3 $\delta$ were amplified by RT-PCR using the primers GCTC GGATCCATGGCGGCTCTGAATGG and TCTGCTCGAGGATCAGTAAGGTTTGCTGTCG, and were subcloned into the pGEX6P-1 vector using Bam HI and Xho I sites. The coding sequence of Etr-1 was amplified by RT-PCR using mouse brain total RNA and subcloned into the pCS2+ MT vector. The preparation of the ACTN1 minigene was described previously [34].

\section{P19 cell culture and cell differentiation}

Embryonic carcinoma P19 cells were cultured as described elsewhere [35]. For neural differentiation, P19 cells were allowed to aggregate in Petri dishes (Falcon) at a seeding density of $1 \times 10^{5}$ cells $/ \mathrm{ml}$ in the presence of $1 \mu \mathrm{M}$ alltrans-retinoic acid (RA, Sigma) in $\alpha$-MEM (Minimum Essential Medium, Sigma) supplemented with 10\% FBS (Fetal Bovine Serum, Sigma). After 4 days of aggregation, cells were dissociated into single cells by $0.25 \%$ trypsinethylenediaminetetraacetic acid (EDTA) (Sigma) solution and replated in a tissue culture dish at a density of 3-6 $\times$ $10^{5}$ cells $/ \mathrm{ml}$. The cells were then allowed to adhere and were cultured in the absence of RA for 10 days. Media were replaced every 48 hours. COS7 and HeLa cells were grown in DMEM (Dulbecco's Modified Eagle's Medium) supplemented with 10\% FBS (Fetal Bovine Serum, Hyclone).

\section{RNA purification and semi-quantitative RT-PCR}

Purification of total RNA from P19 cells was performed using the TRIzol reagent (Invitrogen) after the cells were washed three times with ice-cold PBS. Total RNAs from adult mouse tissues were commercially available (Ambion, Toyobo). cDNA synthesis was performed by SuperScript III (Invitrogen) with oligo-dT primers, using $1 \mu \mathrm{g}$ of total RNA in $20 \mu \mathrm{l}$ reaction mixture. PCR reactions were carried out in $20 \mu \mathrm{l}$ of reaction mixture containing $1 \mu \mathrm{l}$ of template cDNA, $0.1 \mathrm{U}$ of Go Taq Flexi DNA polymerase (Promega), $1 \times$ GO Taq Flexi Buffer, $2.5 \mathrm{mM} \mathrm{MgCl}_{2}, 0.2 \mathrm{mM}$ dNTPs and 4 pmol of each primer. The PCR conditions consisted of an initial denaturation step at $95^{\circ} \mathrm{C}$ for $3 \mathrm{~min}$, followed by a cycle of denaturing at $95^{\circ} \mathrm{C}$ for $30 \mathrm{~s}$, annealing at $60^{\circ} \mathrm{C}$ for $30 \mathrm{~s}$, and extension at $72^{\circ} \mathrm{C}$ for $30 \mathrm{~s}$. The primer names, sequences and number of cycles were as follows: CUGBP2 exon 13, CACTGCCCACTTTGTACAGC, and CUGBP2 exon 15, CTGATCCTAACCCCAGAAGC, with 30 cycles; ACTN1 exon EF1a, CGCCTCTTTCAACCACTTTG, and ACTN1 exon EF2, TCATGATTCGGGCAAACTCT with 27 cycles; and IR exon 10, CCTTC GAGGATTACCTGCAC, and IR exon 12, TGTGCTC CTCCTGACTTGTG, with 32 cycles. $\beta$-Actin was used as an internal control as described previously [34]. PCR products were analyzed in $6 \%$ polyacrylamide gels stained with Ethidium Bromide (EtBr) and visualized in an UVtrans illuminator (Vilber Lourmat). Each RT-PCR experiment was performed more than three times. Quantitative densitometry of the bands was performed using Image Gauge software (Fuji Film).

\section{Western blot analysis}

Western blotting was performed according to a previously described experimental protocol [36]. Cultured P19 cells were washed with ice-cold PBS, collected in $1 \times$ buffer D, and sonicated 3 times for $30 \mathrm{~s}$ on ice. One microgram and $2 \mu \mathrm{g}$ of whole cell lysates were analyzed by $8 \%$ SDS-polyacrylamide gel electrophoresis to detect full-length GAPDH and CUGBP2, respectively. Because the expression level of the R3 $\delta$ isoform was low, $7 \mu$ g of lysate was used in one lane. The positions of the full-length $(56 \mathrm{kDa})$ and $\mathrm{R} 3 \delta$ $(51 \mathrm{kDa})$ isoform were determined with molecular weight markers (Bio-rad). Anti-CUGBP2 (GENWAY, 1:1000) and anti-GAPDH (IMGENEX, 1:1000) were used as primary antibodies. Anti-rabbit IgG-HRP (GE, 1:2000) conjugated antibody was used as the secondary antibody to detect CUGBP2 and R3 $\delta$ proteins. Anti-goat IgG-HRP conjugated antibody (Invitrogen, 1:3000) was used as secondary antibody to detect GAPDH. The experiments shown in Additional file 1: Figure S1 were performed with $1 \mu \mathrm{g}$ of whole cell extracts and anti-myc (CST, 1:1,000) as a primary antibody and anti-rabbit IgG HRP conjugated antibody as a secondary antibody. The membranes were treated using the ECL kit (GE), and the images were analyzed using the LAS-3000 analyzer (Fuji Film). 


\section{Transient transfection experiments}

Transient transfection experiments were carried out as described previously [36]. Transfection was performed using Lipofectamine 2000 (Invitrogen) according to the manufacturer's instructions. Total RNAs were isolated from transfected cells using the TRIzol reagent (Invitrogen). cDNA synthesis was performed in Superscript III (Invitrogen) with the T7 primer. PCR was performed as described above using the IR exon 10 primer CCTGAAGGAGCTGGAGGAG and the IR exon 12 primer ACCGT CACATTCCCAACATC with 25 cycles, and the primers of ACTN1 exon EF1a and ACTN1 exon EF2 with 25 cycles. The PCR products were analyzed in $6 \%$ native polyacrylamide gels stained with $\mathrm{EtBr}$. The images were then visualized in an UV-transilluminator (Vilbar lourman). Each transfection experiment was performed more than three times. Quantitative densitometry of the bands was performed using Image Gauge software (Fuji Film).

\section{Protein expression}

Fragments of CUGBP2 and the R3 $\delta$ isoform were expressed in Escherichia coli strain BL21(DE3) using the pGEX-6P-1 expression plasmid. The two constructs for GST-CUGBP2 and the GST-R3 $\delta$ isoform were grown in M9 minimal medium containing $1 \mathrm{~g} / \mathrm{L}\left[{ }^{15} \mathrm{~N}\right]$ ammonium chloride (Cambridge Isotope Laboratories) as the sole source of nitrogen until an OD600 of 0.6 was reached, followed by induction with $0.1 \mathrm{mM}$ IPTG. The cultured GST-CUGBP2 and GST-R3 $\delta$ cells were allowed to grow for an additional 8 hours at $30^{\circ} \mathrm{C}$ and 6 hours at $25^{\circ} \mathrm{C}$, respectively. The cells were harvested by centrifugation at $4,000 \mathrm{rpm}$ for $20 \mathrm{~min}$. The harvested cell pellets of CUGBP2 and the R3 $\delta$ isoform were resuspended in buffer 1 [50 mM Tris- $\mathrm{HCl}(\mathrm{pH} 8.0)$ and $1 \mathrm{mM} \mathrm{DTT}]$ and buffer 2 [20 mM Tris- $\mathrm{HCl}$ (pH 8.0), $150 \mathrm{mM} \mathrm{NaCl}, 50 \mathrm{mM}$ glycine ethyl ester, $25 \mathrm{mM}$ arginine, $25 \mathrm{mM}$ glutamic acid and $10 \mathrm{mM} \mathrm{DTT}]$, respectively. The cell solutions were lysed by sonication and the lysate was clarified by centrifugation at 15,000 rpm for $20 \mathrm{~min}$ followed by filtration with a $0.45 \mu \mathrm{m}$ membrane.

\section{Purification of CUGBP2 and the R3 $\delta$ isoform}

Protein lysates were loaded onto a Q-Sepharose column and eluted with a stepwise concentration gradient of $100-300 \mathrm{mM} \mathrm{NaCl}$ in $20 \mathrm{mM}$ Tris (pH 8.0) buffer. Fractions containing GST-tagged CUGBP2 were identified using SDS-PAGE. A HiLoad 16/60 Superdex 75 column (GE Healthcare) connected to an AKTA prime plus (GE Healthcare) was equilibrated with $20 \mathrm{mM}$ Tris- $\mathrm{HCl}$ buffer ( $\mathrm{pH}$ 8.0) containing $150 \mathrm{mM} \mathrm{NaCl}$ and $1 \mathrm{mM}$ DTT with a flow rate of $1.0 \mathrm{~mL} / \mathrm{min}$. The sample was concentrated and applied to the column.

Fractions containing GST-tagged CUGBP2 were identified using SDS-PAGE and pooled. CUGBP2 was cleaved from the GST-tagged protein by 40 units of PreScission protease and extensively dialyzed against PreScission protease cleavage buffer [50 mM Tris- $\mathrm{HCl}$ (pH 7.0), $150 \mathrm{mM}$ $\mathrm{NaCl}, 1 \mathrm{mM}$ EDTA and $1 \mathrm{mM} \mathrm{DTT}]$ at $4^{\circ} \mathrm{C}$ for 48 hours. The cleaved proteins were again applied to a gel filtration column. Finally, CUGBP2 was applied to a GST column, and the flow-through fractions were collected. The pure protein solution was dialyzed extensively against a 20 $\mathrm{mM} \mathrm{NH} 4 \mathrm{HCO} 3$ solution and then lyophilized.

The GST-tagged R3 $\delta$ isoform was isolated from sonicated cell extracts with a glutathione Sepharose 4 Fast Flow (GE Healthcare) column according to a standard protocol. The R3 $\delta$ isoform was cleaved from the fusion protein by PreScission protease and the GST-tags were removed with a GST column under the same conditions as those used for CUGBP2. Finally, the R3 $\delta$ isoform was purified by a reverse phase HPLC COSMOSIL C18 column $(10 \mathrm{~mm}$ I.D. $\times 250 \mathrm{~mm}$, Nacalai Tesque Inc.) protected by a guard column. Eluent A was $100 \%$ water with $0.1 \%$ (v/v) trifluoroacetic acid (TFA); eluent B was $99.8 \%$ $(\mathrm{v} / \mathrm{v})$ acetonitrile with $0.1 \%(\mathrm{v} / \mathrm{v})$ TFA. The gradient was as follows: $0 \mathrm{~min}, 5 \% \mathrm{~B} ; 5 \mathrm{~min}, 5 \% \mathrm{~B} ; 70 \mathrm{~min}, 70 \% \mathrm{~B}$. The flow rate was $2 \mathrm{~mL} / \mathrm{min}$. The UV wavelength was $280 \mathrm{~nm}$. Protein fractions from 50-51 min were collected, dialyzed and then lyophilized.

\section{Nuclear magnetic resonance (NMR) spectroscopy}

For NMR measurements, the samples were concentrated to $0.1 \mathrm{mM}$ in $20 \mathrm{mM}$ Bis-Tris $(\mathrm{pH} 7.0)$ containing $100 \mathrm{mM} \mathrm{NaCl}, 1 \mathrm{mM}$ 1,4-DL-dithiothreitol- $d 10$ (d-DTT) and $0.02 \% \mathrm{NaN}_{3}$ (in $90 \% \mathrm{H}_{2} \mathrm{O} / 10 \% \mathrm{D}_{2} \mathrm{O}$ ), using an Amicon Ultra-4 filter (3000 MWCO, Millipore). The NMR experiments were performed for the RNA-free forms and for the RNA-bound forms on an $800 \mathrm{MHz}$ spectrometer (Bruker AVANCE III 800) equipped with a TCI-cryogenic probe at $15^{\circ} \mathrm{C}$. The NMR data were processed using NMRPipe [37]. Analyses of the processed data were performed with the program NMRViewJ [38]. The ${ }^{15} \mathrm{~N}$ chemical shift was calculated by using the ratio, $\gamma_{\mathrm{N}} / \gamma_{\mathrm{H}}=$ 0.101329118 .

\section{Comparative modeling and molecular dynamics simulation}

Comparative modeling was performed in the $\mathrm{PBDj}$ site http://sysimm.ifrec.osaka-u.ac.jp/sfas/. MD simulations were carried out to confirm the structural stability of the RRM3 conformation in CUGBP2 and CUGBP2 R3 8 . Detailed information on these proteins and the MD conditions used is provided in Table 2. The pdb files of prediction and simulation are available as Additional file: Additional file $2 \mathrm{~A}$ is the pdb for the RRM3 of CUGBP2 at $0 \mathrm{~ns}$, Additional file $3: 2 \mathrm{~B}$ shows the pdb for CUGBP2 at $5 \mathrm{~ns}$, Additional file 4: $2 \mathrm{C}$ is the pdb for R3 $\delta$ at 0 ns, and Additional file 5: 2D corresponds to 
Table 2 The conditions of molecular dynamic simulations

\begin{tabular}{ccc}
\hline & CUGBP2 & CUGBP2 R38 \\
\hline \# Total atoms & 39883 & 28754 \\
\# Protein atoms & 1402 & 1131 \\
\# Sol water & 12821 & 9205 \\
\# lons & 3 & 4 \\
\hline
\end{tabular}

the pdb for $\mathrm{R} 3 \delta$ at $5 \mathrm{~ns}$. MD simulations were carried out by using the AMBER10 program package with force field 03 [39]. Simulation time was $5 \mathrm{~ns}(\Delta \mathrm{t}=1 \mathrm{fs})$ at room temperature $(300 \mathrm{~K})$ with periodic boundary conditions.

\section{Additional material}

Additional file 1: Figure S1. Transient transfection of the effectors with the ACTN1 minigene or the IR minigene. Transient transfection experiments with the ACTN 1 minigene (upper panel) or the $I R$ minigene (lower panel) were performed as shown in Figure 3. Whole cell extracts were analyzed by western blot analysis using an anti-Myc or anti-GAPDH antibody. Endogenous expression of GAPDH and over-expressing effectors containing CUGBP2, R3 $\delta$, and Etr-1 were observed.

Additional file 2: Additional file 1A. The pdb file of the predicted CUGBP2 RRM3 at 0 ns.

Additional file 3: Additional file 1B. The pdb file of the simulated CUGBP2 RRM3 at $5 \mathrm{~ns}$

Additional file 4: Additional file 1C. The pdb file of the predicted structure, which contains the partial RRM3 and linker region in CUGBP2 $\mathrm{R} 3 \delta$, at 0 ns.

Additional file 5: Additional file 1D. The pdb file of the simulated structure, which contains the partial RRM3 and linker region in CUGBP2 $\mathrm{R} 3 \delta$, at 5 ns.

\section{Acknowledgements}

We thank Dr. Webster at UCSD for providing the IR minigene (pIRb). We thank Dr. Muto at RIKEN GSC for providing the chemical shift table of CUGBP1. Bioedit Itd. carried out English correction, including comprehensive editing. This research was supported in part by a Grant-in-Aid for Scientific Research from the Japan Society for the Promotion of Science of Japan (to HS) and in part by Intramural Research Grant (22 - 5) for Neurological and Psychiatric Disorders of NCNP.

\section{Author details}

${ }^{1}$ Center for Nano Materials and Technology, Japan Advanced Institute of Science and Technology, Ishikawa 923-1292, Japan. ${ }^{2}$ School of Materials Science, Japan Advanced Institute of Science and Technology, Ishikawa 9231292, Japan. ${ }^{3}$ School of Knowledge Science, Japan Advanced Institute of Science and Technology, Ishikawa 923-1292, Japan. ${ }^{4}$ Japan Science and Technology Agency, ERATO, Shimoda Nano-Liquid Process Project, 2-5-3 Asahidai, Nomi, Ishikawa 923-1211, Japan. ${ }^{5}$ Department of Pharmacy, University of Rajshahi, Rajshahi 6205, Bangladesh.

\section{Authors' contributions}

HS carried out the design of the study, helped to perform the transient transfection assay, and drafted the manuscript. MT carried out the NMR experiment and helped to draft the manuscript. AS carried out the MD study and helped to draft the manuscript. AKA carried out expression analysis and transient transfection assays. LTV participated in expression analysis and transient transfection assays. YS carried out plasmid construction for the NMR experiment and transient transfection assays. HCD participated in the MD study. SO participated in the NMR experiment and helped to the draft manuscript. ПT participated in the design of the study and helped to draft the manuscript. All authors read and approved the final manuscript.

Received: 20 September 2011 Accepted: 20 March 2012

Published: 20 March 2012

\section{References}

1. Philips AV, Timchenko LT, Cooper TA: Disruption of Splicing Regulated by a CUG-Binding Protein in Myotonic Dystrophy. Science 1998, 280:737-741.

2. Kim-Ha J, Kerr K, Macdonald PM: Translational regulation of oskar mRNA by bruno, an ovarian RNA-binding protein, is essential. Cell 1995, 81:403-412.

3. Ladd AN, Charlet BN, Cooper TA: The CELF family of RNA binding proteins is implicated in cell-specific and developmentally regulated alternative splicing. Mol Cell Biol 2001, 21:1285-1296.

4. Choi DK, Ito T, Tsukahara F, Hirai M, Sakaki Y: Developmentally regulated expression of mNapor encoding an apoptosis-induced ELAV-type RNA binding protein. Gene 1999, 237:135-142.

5. Good PJ, Chen Q, Warner SJ, Herring D: A family of human RNA-binding proteins related to the Drosophil Bruno translational regulator. J Biol Chem 2000, 275:28583-28592.

6. Barreau C, Paillard L, Mereau A, Osborne HB: Mammalian CELF/Bruno-like RNA-binding proteins: molecular characteristics and biological functions. Biochimie 2006, 88:515-525

7. Timchenko LT, Miller JW, Timchenko NA, DeVore DR, Datar KV, Lin L, Roberts R, Caskey CT, Swanson MS: Identification of a (CUG)n triplet repeat RNA-binding protein and its expression in myotonic dystrophy. Nucleic Acids Res 1996, 24:4407-4414.

8. Poleev A, Hartmann A, Stamm S: A trans-acting factor, isolated by the three-hybrid system, that influences alternative splicing of the amyloid precursor protein minigene. Eur J Biochem 2000, 267:4002-4010.

9. Mankodi A, Takahashi MP, Jiang H, Beck CL, Bowers WJ, Moxley RT, Cannon SC, Thornton CA: Expanded CUG repeats trigger aberrant splicing of $\mathrm{ClC}-1$ chloride channel pre-mRNA and hyperexcitability of skeletal muscle in myotonic dystrophy. Mol Cell 2002, 10:35-44.

10. Charlet-B N, Savkur RS, Singh G, Philips AV, Grice EA, Cooper TA: Loss of the muscle specific chloride channel in type 1 myotonic dystrophy due to misregulated alternative splicing. Mol Cell 2002, 10:45-53.

11. Suzuki H, Jin Y, Otani H, Yasuda K, Inoue K: Regulation of alternative splicing of a-actinin transcript by Bruno-like proteins. Genes Cells 2002, 7:133-141.

12. Gromak N, Matlin AJ, Cooper TA, Smith CW: Antagonistic regulation of aactinin alternative splicing by CELF proteins and polypyrimidine tract binding protein. RNA 2003, 9:443-456

13. Savkur RS, Philips AV, Cooper TA: Aberrant regulation of insulin receptor alternative splicing is associated with insulin resistance in myotonic dystrophy. Nat Genet 2001, 29:40-47.

14. Dansithong W, Paul S, Comai L, Reddy S: MBNL1 is the primary determinant of focus formation and aberrant insulin receptor splicing in DM1. J Biol Chem 2005, 280:5773-5780.

15. Singh G, Charlet BN, Han J, Cooper TA: ETR-3 and CELF4 protein domains required for RNA binding and splicing activity in viv. Nucleic Acids Res 2004, 32:1232-1241.

16. Zhang W, Liu H, Han K, Grabowski PJ: Region-specific alternative splicing in the nervous system: implications for regulation by the RNA-binding protein NAPOR. RNA 2002, 8:671-685.

17. Han J, Cooper TA: Identification of CELF splicing activation and repression domains in viv. Nucleic Acid Res 2005, 33:2769-2780.

18. Paillard L, Omillia F, Legagneux V, Bassez T, Maniey D, Osborne HB: EDEN and EDEN-BP, a cis element and an associated factor that mediate sequence-specific mRNA deadenylation in Xenopus embryos. EMBO $\lrcorner$ 1998, 17:178-287.

19. Hashimoto $Y$, Suzuki H, Kageyama $Y$, Yasuda $K$, Inoue $K$ : Bruno-like protein is localized to zebrafish germ plasm during the early cleavage stages. Gene Expr Patterns 2006, 6:201-205.

20. Hashimoto Y, Maegawa S, Nagai T, Yamaha E, Suzuki H, Yasuda K, Inoue K: Localized maternal factors are required for zebrafish germ cell formation. Dev Biol 2004, 268:152-161.

21. Thimchenko NA, Welm AL, LU X, Thimchenko LT: CUG repeat binding protein (CUGBP1) interacts with the $5^{\prime}$ region of C/EBPbeta mRNA and 
regulates translation of C/EBPbeta isoforms. Nucleic Acids Res 1999, 27:4517-4525.

22. Tsuda K, Kuwasako K, Takahashi M, Someya T, Inoue M, Terada T, Kobayashi N, Shirouzu M, Kigawa T, Tanaka A, Sugano S, Güntert P, Muto Y, Yokoyama S: Structural basis for the sequence-specific RNA-recognition mechanism of human CUG-BP1 RRM3. Nucleic Acids Res 2009, 37:5151-5166.

23. Burd CG, Dreyfuss $G$ : Conserved structures and diversity of functions of RNA-binding proteins. Science 1994, 265:615-621.

24. Auweter SD, Fasan R, Reymond L, Underwood JG, Black DL, Pitsch S, Allain FH: Molecular basis of RNA recognition by the human alternative splicing factor Fox-1. EMBO J 2006, 25:163-173.

25. Kellenberger E, Stier G, Sattler M: Induced folding of the U2AF35 RRM upon binding to U2AF65. FEBS Lett 2002, 528:171-176.

26. Kielkopf CL, Lücke S, Green MR: U2AF homology motifs: protein recognition in the RRM world. Genes Dev 2004, 18:1513-1526.

27. Ladd AN, Cooper TA: Multiple domains control the subcellular localization and activity of ETR-3, a regulator of nuclear and cytoplasmic RNA processing events. J Cell Sci 2004, 117:3519-3529.

28. Kosaki A, Nelson J, Webster NJG: Identification of intron and exon sequences involved in alternative splicing of insulin receptor Pre-mRNA. J Biol Chem 1998, 273:10331-10337.

29. Sen S, Talukdar I, Liu Y, Tam J, Reddy S, Webster NJ: Muscleblind-like 1 (Mbnl1) promotes insulin receptor exon 11 inclusion via binding to a downstream evolutionarily conserved intronic enhancer. J Biol Chem 2010, 285:25426-25437.

30. ElAntak L, Tzakos AG, Locker N, Lukavsky PJ: Structure of elF3b RNA recognition motif and its interaction with elF3j: structural insights into the recruitment of elF3b to the $40 \mathrm{~S}$ ribosomal subunit. J Biol Chem 2007, 282:8165-8174.

31. Fleming K, Ghuman J, Yuan X, Simpson P, Szendröi A, Matthews S, Curry S: Solution structure and RNA interactions of the RNA recognition motif from eukaryotic translation initiation factor 4B. Biochemistry 2003, 42:8966-8975

32. Teplova M, Yuan YR, Phan AT, Malinina L, llin S, Teplov A, Patel DJ: Structural basis for recognition and sequestration of $\mathrm{UUU}(\mathrm{OH}) 3^{\prime}$ temini of nascent RNA polymerase III transcripts by La, a rheumatic disease autoantigen. Mol Cell 2006, 21:75-85.

33. Sen S, Talukdar I, Webster NJ: SRp20 and CUG-BP1 modulate insulin receptor exon 11 alternative splicing. Mol Cell Biol 2009, 29:871-880.

34. Alam AH, Suzuki H, Tsukahara T: Retinoic acid treatment and cell aggregation independently regulate alternative splicing in P19 cells during neural differentiation. Cell Biol Int 2010, 34:631-643.

35. Komatsu M, Kominami E, Arahata K, Tsukahara T: Cloning and characterization of two neural-salient serine/arginine-rich (NSSR) proteins involved in the regulation of alternative splicing in neurons. Genes Cells 1999, 4:593-606.

36. Hakim NH, Kounishi T, Alam AH, Tsukahara T, Suzuki H: Alternative splicing of Mef2c promoted by Fox-1 during neural differentiation in P19 cells. Genes Cells 2010, 15:255-267.

37. Delaglio F, Grzesiek S, Vuister GW, Zhu G, Pfeifer J, Bax A: NMRPipe: A multidimensional spectral processing system based on UNIX pipes. J Biomol NMR 1995, 6:277-293.

38. Johnson BA: Using NMRView to visualize and analyze the NMR spectra of macromolecules. Methods Mol Biol 2004, 278:31.

39. Cornell WD, Cieplak P, Bayly Cl, Gould IR, Merz KM, Ferguson DM, Spellmeyer DC, Fox T, Caldwell JW, Kollman PA: A Second Generation Force Field for the Simulation of Proteins, Nucleic Acids, and Organic Molecules. J Am Chem Soc 1995, 117:5179-5197.

doi:10.1186/1471-2091-13-6

Cite this article as: Suzuki et al.: Alternative splicing produces structural and functional changes in CUGBP2. BMC Biochemistry 2012 13:6.

\section{Submit your next manuscript to BioMed Central and take full advantage of:}

- Convenient online submission

- Thorough peer review

- No space constraints or color figure charges

- Immediate publication on acceptance

- Inclusion in PubMed, CAS, Scopus and Google Scholar

- Research which is freely available for redistribution 